\title{
Alteration of the PKC-mediated signaling pathway for smooth muscle contraction in obstruction-induced hypertrophy of the urinary bladder
}

\author{
Shaohua Chang ${ }^{1}$, Joseph A Hypolite', Sunish Mohanan', Stephen A Zderic ${ }^{1,2}$, Alan J Wein ${ }^{1}$ \\ and Samuel Chacko ${ }^{1,3}$
}

\begin{abstract}
Normal urinary bladder function requires contraction and relaxation of the detrusor smooth muscle (DSM). The DSM undergoes compensatory hypertrophy in response to partial bladder outlet obstruction (PBOO) in both men and animal models. Following bladder hypertrophy, the bladder either retains its normal function (compensated) or becomes dysfunctional (decompensated) with increased voiding frequency and decreased void volume. We analyzed the contractile characteristics of DSM in a rabbit model of PBOO. The protein kinase C (PKC) agonist phorbol 12, 13-dibutyrate (PDBu) elicited similar levels of contraction of DSM strips from normal and compensated bladders. However, PDBu-induced contraction decreased significantly in DSM strips from decompensated bladders. The expression and activity of PKC- $\alpha$ were also lowest in decompensated bladders. The PKC-specific inhibitor bisindolylmaleimide-1 (Bis) blocked PDBu-induced contraction and PKC activity in all three groups. Moreover, the phosphorylation of the phosphoprotein inhibitor CPI-17 (a 17-kDa PKC-potentiated inhibitory protein of protein phosphatase-1) was diminished in DSM from the decompensated bladder, which would result in less inhibitory potency of CPI-17 on myosin light chain phosphatase activity and contribute to less contractility. Immunostaining revealed the colocalization of PKC and phosphorylated $\mathrm{CPI}-17$ in the DSM and confirmed the decreases of these signaling proteins in the decompensated bladder. Our results show a differential PKC-mediated DSM contraction with corresponding alterations of PKC expression, activity and the phosphorylation of $\mathrm{CPI}-17$. Our finding suggests a significant correlation between bladder function and PKC pathway. An impaired PKC pathway appears to be correlated with severe bladder dysfunction observed in decompensated bladders. Laboratory Investigation (2009) 89, 823-832; doi:10.1038/labinvest.2009.38; published online 20 April 2009
\end{abstract}

KEYWORDS: bladder outlet obstruction; CPI-17; PDBu-induced contraction; PKC

Partial bladder outlet obstruction (PBOO) is caused by benign prostatic hyperplasia $(\mathrm{BPH})$. PBOO results in an impaired ability of the urinary bladder to store and empty urine. $\mathrm{PBOO}$ in a rabbit model results in bladder dysfunction and in alterations of the mechanistic steps involved in detrusor smooth muscle (DSM) contractility, similar to those found in men with $\mathrm{BPH} .{ }^{1,2}$ In response to $\mathrm{PBOO}$, the DSM undergoes compensatory hypertrophy to produce the increased force necessary to expel urine against an obstruction. In some rabbits, bladder wall hypertrophy and the associated remodeling of the DSM is sufficient to maintain nearly normal bladder function, whereas other rabbits develop severe bladder dysfunction, resulting in changes in voiding frequency, void volume and residual volume. ${ }^{3,4}$ Interestingly, BPH-induced DSM hypertrophy in men also produces either nearly normal or severely defective bladder function.

Several studies have described the alterations in DSM contractility and expression of contractile and regulatory proteins after 2 weeks of PBOO. Metabolic cage monitoring of bladder function following PBOO revealed a decreased void volume and increased voiding frequency, consistent with overexpression of the N-terminal myosin isoform SM-A (low ATPase, compared to the SM-B isoform) and less smooth muscle myosin heavy chain. ${ }^{5}$ Besides the change of contractile protein in DSM, PBOO also induces a change of

\footnotetext{
Division of Urology, Department of Surgery, University of Pennsylvania, Glenolden, PA, USA; ${ }^{2}$ The Children's Hospital of Philadelphia, Philadelphia, PA, USA and ${ }^{3}$ Department of Pathobiology, University of Pennsylvania, Philadelphia, PA, USA Correspondence: Dr S Chacko, DVM, PhD, Glenolden Research Laboratory, University of Pennsylvania, 500 S Ridgeway Avenue, Glenolden, PA 19036, USA. E-mail: Samuel.Chacko@uphs.upenn.edu 
regulatory protein, such as Rho-kinase ${ }^{3}$ and sarcoplasmic reticulum proteins. ${ }^{6,7}$ Protein kinase $\mathrm{C}$ (PKC) is another important regulatory protein in smooth muscle contractility. ${ }^{8-10}$ Phorbol 12, 13-dibutyrate (PDBu) induces rabbit DSM contraction by activating PKC. ${ }^{11,12}$ The activated PKC can phosphorylate a $17-\mathrm{kDa}$ protein called PKCpotentiated inhibitory protein of protein phosphatase-1 (CPI-17). ${ }^{13}$ Phosphorylation of CPI-17 at Thr-38 by PKC produces over a 1000 -fold increase in the inhibitory potency of CPI-17. Thus, phosphorylated CPI-17 inhibits myosin phosphatase activity, ${ }^{14}$ leading to increased phosphorylation of myosin light chain and contraction of smooth muscle. ${ }^{15}$ A recent study shows that $\mathrm{PBOO}$ selectively abolishes PKCinduced contraction of rabbit DSM. ${ }^{16}$

This study is designed to evaluate whether there is a differential PKC-induced contraction in DSM from compensated and decompensated bladders and whether this change is related with PKC function including PKC expression, activity and CPI-17 phosphorylation. Our results demonstrate that there is no significant change on PKC-induced contraction and PKC function in compensated bladder compared with control. However, decompensated bladders generate significantly less PKC-induced contraction that is accompanied by a significant decrease in PKC expression, PKC activity and CPI-17 phosphorylation compared with control. This finding suggests that the downregulation of PKC signaling is one of the underlying molecular mechanism involved in the transition from compensated to decompensated.

\section{MATERIALS AND METHODS}

\section{Partial Bladder Outlet Obstruction}

The protocol for animal experiments was approved by the University of Pennsylvania's Institutional Animal Care Use Committee. The surgical procedure to create $\mathrm{PBOO}$ was described in our previous publication. ${ }^{6}$ Adult male New Zealand white rabbits weighing approximately $3 \mathrm{~kg}$ were anesthetized with ketamine/xylazine $(25 \mathrm{mg} / 10 \mathrm{mg} ;$ i.m.). Surgical anesthesia was maintained with pentobarbital $(25 \mathrm{mg} / \mathrm{kg}$; i.v.). Under sterile conditions, the urinary bladder was catheterized with an 8-French Foley catheter, and the bladder was exposed through a midline incision. PBOO was initiated by placing a 0 -silk ligature around the catheterized urethra. The catheter was removed and the incision was closed with a 3-0 silk. After PBOO, rabbits were kept for 2 weeks and then killed for bladder tissue collection. One day before killing, rabbits were placed in metabolic cages with free access to food and water to monitor bladder voiding patterns. Frequency was determined as voids per $24 \mathrm{~h}$. Average voided volume is expressed as ml per void. Selection criteria for decompensated bladders include greater than 30 voids per day and an average voided volume of less than $4 \mathrm{ml}$. On the other hand, the compensated group had less than 30 voids per day and an average voided volume of more than
Table 1 PBOO induced changes on voiding pattern

\begin{tabular}{|c|c|c|c|}
\hline & Normal & Compensated & Decompensated \\
\hline $\begin{array}{l}\text { Voiding frequency } \\
\text { (voids per } 24 \mathrm{~h} \text { ) }\end{array}$ & $4 \pm 3$ & $16 \pm 8$ & $43 \pm 13$ \\
\hline $\begin{array}{l}\text { Voided volumes (ml } \\
\text { per void) }\end{array}$ & $26.0 \pm 16.0$ & $8.7 \pm 4.5$ & $2.5 \pm 1.0$ \\
\hline
\end{tabular}

$4 \mathrm{ml}^{7}$ Analyzed metabolic cage data for animals in two groups are shown in Table 1.

\section{Force Measurements}

Bladder smooth muscle strips were prepared by removing the mucosal and serosal layers from the middle bladder body. Longitudinal muscle strips $(3 \times 10 \mathrm{~mm})$ were suspended in $15 \mathrm{ml}$ of Tyrode's buffer $(124.9 \mathrm{mM} \mathrm{NaCl}, 2.5 \mathrm{mM} \mathrm{KCl}$, $23.8 \mathrm{mM} \mathrm{NaHCO} 3,0.5 \mathrm{mM} \mathrm{MgCl}_{2}, 0.4 \mathrm{mM} \mathrm{NaH} \mathrm{PO}_{4}$, $1.8 \mathrm{mM} \mathrm{CaCl}_{2}$ and $5.5 \mathrm{mM}$ dextrose) in organ bath (Radnoti) to equilibrate for $1 \mathrm{~h}$ in $95 \% \mathrm{O}_{2}$ and $5 \% \mathrm{CO}_{2}$. Grass Model 7D Polygraph was used to record force generation. The length of optimal force development was determined by increasing the length of each strip in $1.5 \mathrm{~mm}$ increments until maximal contractile force to electrical field stimulation was achieved. ${ }^{17,18} \mathrm{Next}$, the force generation profile in response to $125 \mathrm{mM} \mathrm{KCl}$ was determined. After strips were washed three times with buffer, increasing concentrations of PDBu (0.03, $0.1,0.3,1$ and $3 \mu \mathrm{M}$; Calbiochem) were added and the response to $1 \mu \mathrm{M}$ PDBU was recorded. Next, the specific PKC inhibitor bisindolylmaleimide (Bis) I (10 $\mu \mathrm{M}$; Calbiochem) was preincubated with muscle strips for $20 \mathrm{~min}$ before the addition of $\mathrm{PDBu}$ to confirm that the PDBu-induced contraction is mediated by PKC.

Tissue Preparation for the Measurement of PKC Activity Muscle strips were placed in organ baths containing Tyrode's buffer equilibrated with $95 \% \mathrm{O}_{2}$ and $5 \% \mathrm{CO}_{2}$ at $37^{\circ} \mathrm{C}$. Individual strips were snap-frozen in liquid nitrogen for $30 \mathrm{~s}$, $1 \mathrm{~min}$ or $3 \mathrm{~min}$ after the addition of $3 \mu \mathrm{M} \mathrm{PDBu}$. Each strip in a second set of experiment was first preincubated with $10 \mu \mathrm{M}$ Bis for $20 \mathrm{~min}$ and then snap-frozen in liquid nitrogen for $30 \mathrm{~s}, 1 \mathrm{~min}$ or $3 \mathrm{~min}$ after the addition of $3 \mu \mathrm{M}$ PDBu. Untreated strips were used as control. Frozen tissues were saved in liquid nitrogen for subsequent PKC assay.

\section{PKC Assay}

PKC activity was measured using the PepTag Assay kit from Promega, according to the manufacturer's protocol. PepTag C1 peptide is a brightly colored, fluorescent peptide substrate of PKC. Phosphorylation of $\mathrm{C} 1$ peptide by PKC alters its charge from +1 to -1 . On an agarose gel, the phosphorylated $\mathrm{C} 1$ migrated toward the positive electrode and the nonphosphorylated $\mathrm{C} 1$ toward the negative electrode. 
Tissue extracts for PKC analysis were prepared by homogenizing tissue in cold PKC extraction buffer. The supernatant was collected after centrifuging the lysate for $5 \mathrm{~min}$ at $14000 \mathrm{~g}$. A PKC assay standard was prepared by diluting purified PKC enzyme in dilution buffer. For each sample, the assay mix consisted of reaction buffer, $\mathrm{C} 1$ peptide and activation buffer. After adding sample or standard to the assay mix, the reaction tubes were incubated at $30^{\circ} \mathrm{C}$ for $30 \mathrm{~min}$. The reaction was stopped by placing the reaction tubes in a boiling water bath for $10 \mathrm{~min}$. The samples were then ready to load onto the gel.

\section{Protein Extraction and Western Blot Analysis}

Total protein was extracted from frozen bladder tissue ( $\sim 50 \mathrm{mg}$ ) using $1 \mathrm{ml}$ extraction buffer, which contained $50 \mathrm{mM}$ Tris (pH 6.8), 20\% glycerol, $1 \%$ SDS and protease inhibitors $(0.8 \mathrm{mM}$ PMSF, $10 \mu \mathrm{M}$ pepstatin, $1 \mu \mathrm{M}$ antipain and $0.1 \mathrm{mg} / \mathrm{ml}$ trypsin inhibitor). Protein concentration was determined by DC protein assay kit (Bio-Rad). Equal amounts of total protein $(20 \mu \mathrm{g})$ were separated on $14 \%$ SDS-PAGE gels and transferred to a PVDF membrane (Millipore, Bedford, MA, USA) overnight at $30 \mathrm{~V}$ (Bio-Rad mini-transfer unit). After blocking with $5 \%$ fat-free milk in PBS (phosphate-buffered saline) for $1 \mathrm{~h}$, the membrane was incubated with primary antibodies, including mouse monoclonal PKC- $\alpha$ (from ABR Affinity BioReagents; 1:2000 dilution), goat polyclonal CPI-17 and goat polyclonal phospho-CPI-17 (Thr-38) (from Santa Cruz; 1:2000 dilution) and mouse monoclonal $\alpha$-actin (Sigma; 1:5000 dilution) for $2 \mathrm{~h}$ at room temperature. The membrane was then washed and incubated with secondary horseradish peroxidase-linked anti-mouse antibody or anti-goat antibody (1:5000 dilution) for $1 \mathrm{~h}$. Membranes were washed thoroughly with PBS containing 0.05\% Tween 20 (Sigma) between incubations. Target proteins were detected using an enhanced chemiluminescence (ECL) kit from Amersham Life Sciences. The amounts of PKC, CPI-17 and phosphor-CPI-17 were determined by reflectance scanning densitometry using Bio-Rad GS-800 Calibrated Densitometer and Quantity 1 software (Bio-Rad). Smooth muscle $\alpha$-actin was used as an internal control to normalize western blot data.

\section{Immunohistochemistry}

Cross sections $(5 \mu \mathrm{M})$ of bladder from sham and $\mathrm{PBOO}$ rabbits were made from paraffin blocks. Tissue sections were deparaffinized, put into descending grades of alcohol (100, $95,70,30 \%$ and $1 \times$ PBS) and blocked $30 \mathrm{~min}$ in $1 \%$ BSA solution. After blocking, sections were incubated with antibodies (described above) specific for PKC- $\alpha /$ CPI-17 (PKC- $\alpha$ and CPI-17 were used for double staining on the same section) and phosphor-CPI-17 (a serial section from the same block). For each antibody, the incubation time is $2 \mathrm{~h}$ at room temperature and the dilution is 1:200. Sections were washed three times in PBS after primary antibody incubation and treated with secondary antibodies (anti-mouse FITC, anti- goat cy3 and anti-goat FITC; Sigma) at a dilution of 1:400 for $1 \mathrm{~h}$. Sections were washed again three times with PBS and mounted with a drop of mounting medium (Aqua-Mount; Lerner Labs, Pittsburgh, PA, USA). Sections were viewed under Olympus Confocal Laser Scanning Biological Microscope. Images were captured using FluoView FV1000 software. Negative controls to confirm the specificity of immunostaining were prepared using mouse or goat serum in place of the primary antibodies.

\section{Statistical Analysis}

All data are expressed as the mean plus or minus standard error of the mean (s.e.m.) and analyzed by one-way ANOVA. Two types of PBOO were compared with control by Dunnett's test, $P<0.05$ considered statistically significantly. Each $n$ refers to a set of normal, compensated and decompensated rabbits.

\section{RESULTS \\ Compensated vs Decompensated Bladder Function Following PBOO}

$\mathrm{PBOO}$ induces detrusor hypertrophy and alters bladder function. Although characterization of function after PBOOinduced DSM hypertrophy as compensated and decompensated is difficult, some bladders maintain nearly normal function (compensated) whereas others show severe dysfunction, including increased urinary frequency and decreased void volume (decompensated). In normal rabbit, the average voiding volume and frequency are $\sim 26 \mathrm{ml}$ and $\sim 4$ voids per day respectively. PBOO can increase the frequency to $\sim 43$ voids per day and reduce the volume to $\sim 2.5 \mathrm{ml}$. Figure 1 shows a distinct distribution of obstructed rabbits. One group has lower urinary frequency (less than 30 voids per day) and high voiding volume, which is considered as compensated. The other group has high frequency (more than 30 voids per day) and low voiding volume (less than $2.5 \mathrm{ml}$ per void), which is considered as decompensated. We have noticed a difference in PKC expression and activity between the compensated and decompensated bladders as shown below.

\section{PBOO-Induced Differential DSM Contraction in Response to $\mathrm{KCl}$ and PDBu}

The compensated and decompensated bladder function after $\mathrm{PBOO}$ suggested a corresponding alteration in DSM contractility. We measured DSM contraction in response to $125 \mathrm{mM} \mathrm{KCl}$, which directly caused muscle contraction by depolarized DSM membrane. DSM strips from compensated bladders produced similar force compared with normal, whereas DSM strips from decompensated bladders generated much less force compared to normal controls (Figure 2b). The decreased $\mathrm{KCl}$-induced contraction in decompensated DSM may be due to decreased smooth muscle myosin heavy chain. Our previous study shows a significantly decreased expression of smooth muscle myosin heavy chain mRNA $\left(1.57 \pm 0.19 \times 10^{8}\right.$ copies per $100 \mathrm{ng}$ total RNA), compared 


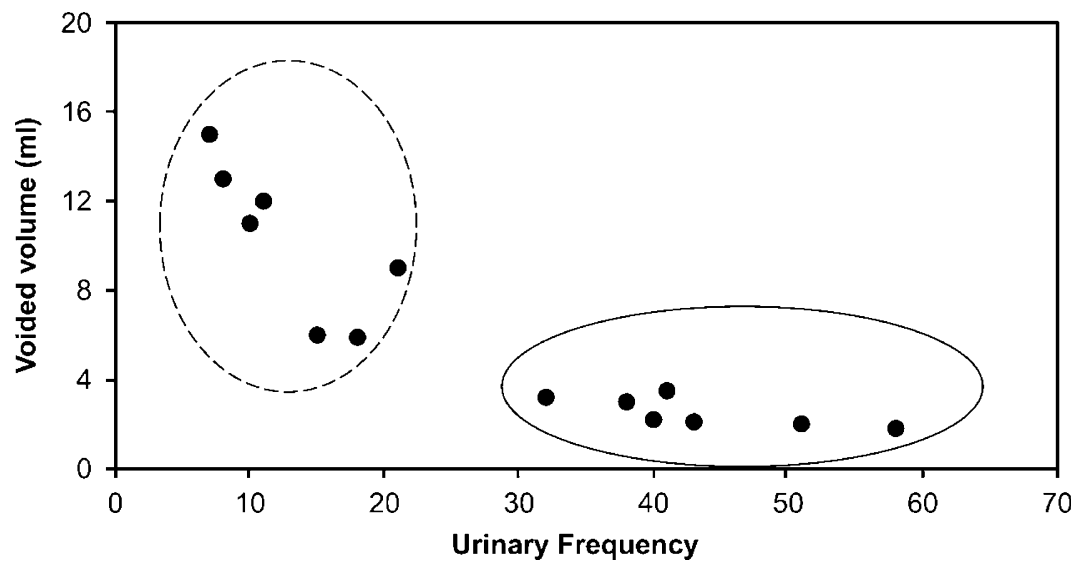

Figure 1 PBOO altered rabbit voiding frequency and volume per void. The obstructed animals were plotted by voiding volume and urinary frequency per void. The distribution shows two distinct groups: one group with high volume and low frequency (compensated) and the other group with low volume and high frequency (decompensated).

a
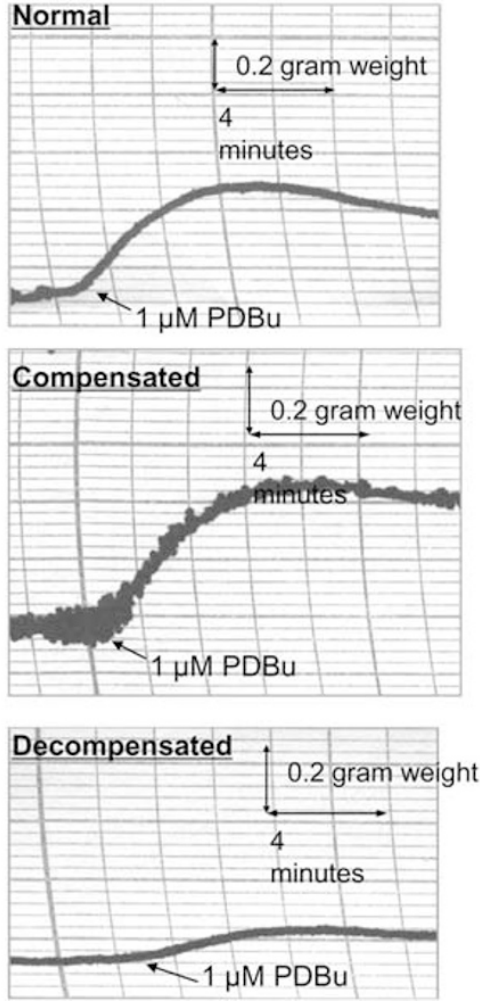

b

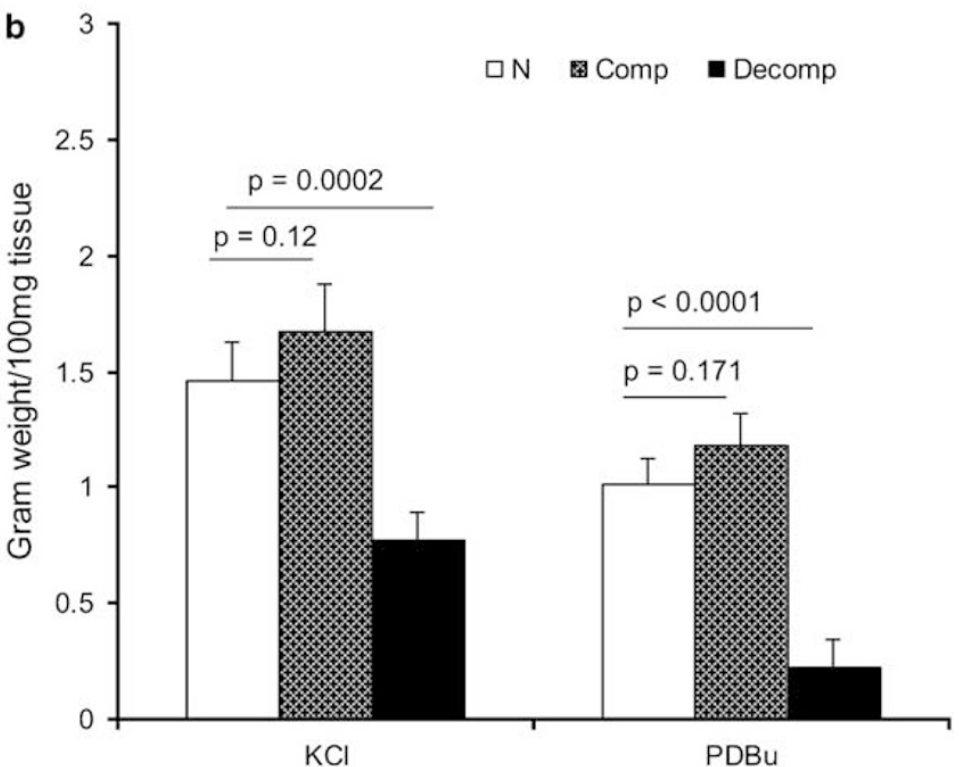

Figure 2 PBOO-induced differential alteration of DSM contraction. (a) Representative force generation profiles of DSM strips from normal, compensated and decompensated bladders in response to $1 \mu \mathrm{M}$ PDBu (two horizontal boxes $=4 \mathrm{~min}$, five small vertical boxes $=0.2 \mathrm{gram}$ weight). (b) Analyzed force generation from four experiments ( $n=4$ animals per group) in response to $125 \mathrm{mM} \mathrm{KCl}$ and $1 \mu \mathrm{M} \mathrm{PDBu}$. Force is normalized by the weight of muscle strips. There is a significant decrease of DSM contractility in decompensated bladder.

with sham control $\left(2.04 \pm 0.15 \times 10^{8}\right.$ copies per $100 \mathrm{ng}$ total RNA). ${ }^{5}$ This result indicates that the alteration of contractile protein in decompensated DSM causes impaired DSM contractility despite smooth muscle hypertrophy after PBOO.

Besides the contractile proteins, regulatory proteins also have an important function in DSM contractility. We further examined the PKC-mediated DSM contraction by giving a PKC agonist, PDBu. Figure $2 \mathrm{a}$ shows the representative force profiles of DSM from normal, compensated and decompensated bladders in response to $1 \mu \mathrm{M}$ PDBU. DSM from normal and compensated bladders produced similar levels of force, whereas $\mathrm{PDBu}-$ 


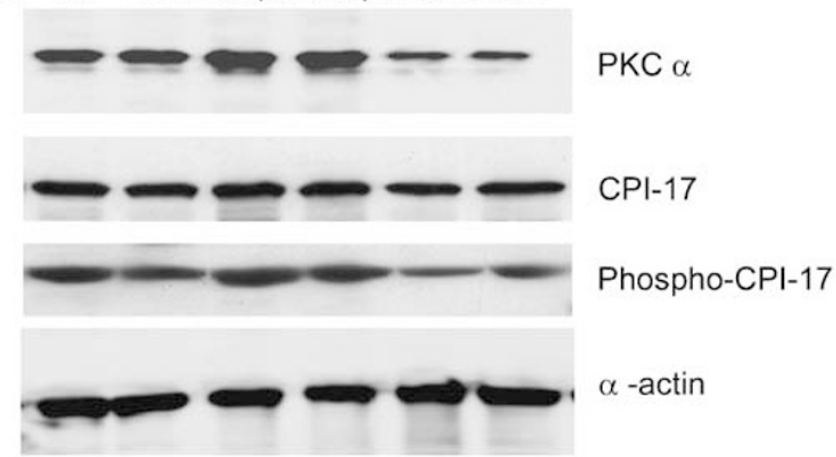

b

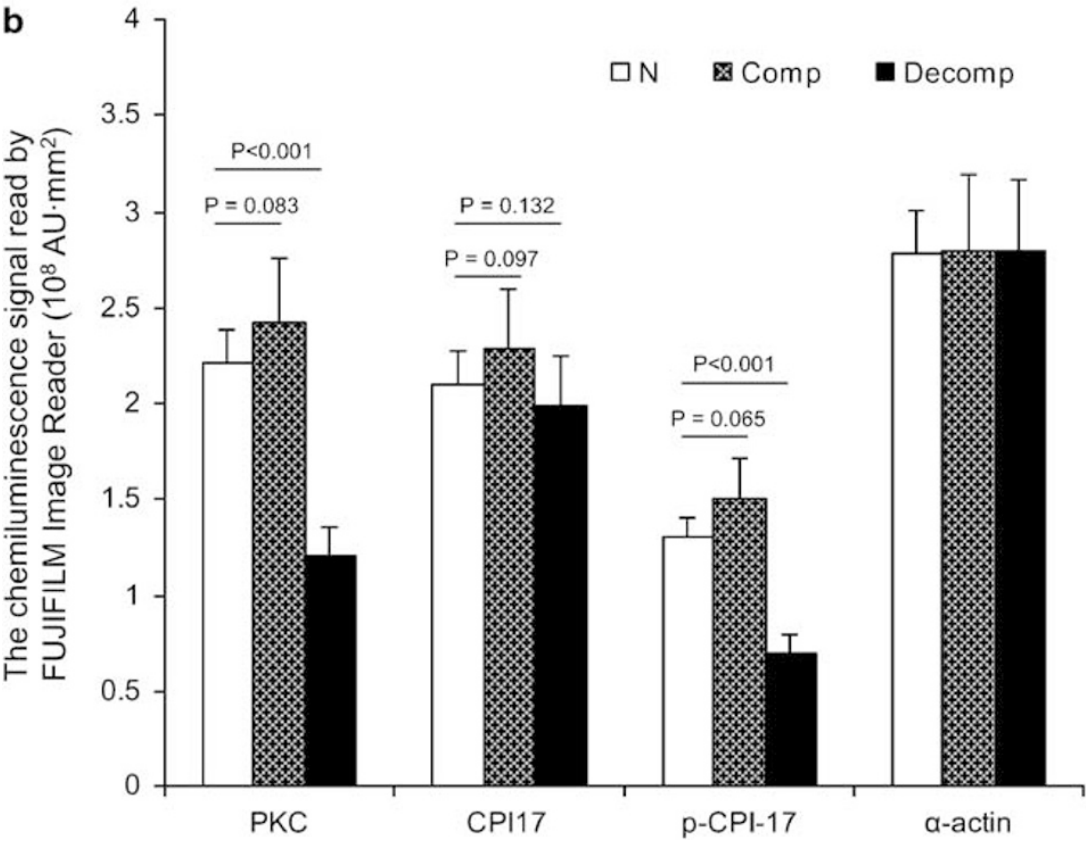

Figure 3 Alteration in the expression of PKC- $\alpha$ and the phosphorylation of CPI-17. (a) Representative western blots for PKC- $\alpha$, CPI-17, phosphorylated CPI-17 (phospho-CPI-17) and smooth muscle $\alpha$-actin (used as an internal control). Western blots from normal (lanes 1 and 2), compensated (lanes 3 and 4) and decompensated (lanes 5 and 6) bladders are shown. (b) Quantification analysis of ECL signal that represents the amount of target protein ( $n=6$ animals per group). PKC- $\alpha$ expression and phospho-CPI-17 were significantly $(P<0.001)$ decreased in the decompensated bladder DSM.

induced contraction was remarkably decreased in DSM from decompensated bladders. Figure $2 \mathrm{~b}$ shows the analyzed result of DSM contractility in response to $\mathrm{KCl}$ and $\mathrm{PDBu}$. The contractility was significantly decreased in DSM from decompensated bladders compared to normal or compensated groups $(n=4, P<0.01)$. There is about $50 \%$ decrease in $\mathrm{KCl}$-induced contraction in decompensated bladder. Interestingly, the DSM from these bladders showed about $80 \%$ decrease in PDBu-induced contraction. This result suggests an impaired PKC signaling in decompensated DSM, which leads to a larger decrease in $\mathrm{PDBu}$-induced contraction than that seen in $\mathrm{KCl}-$ induced contraction. Preincubation with Bis, a PKC inhibitor, abolished PDBuinduced contraction in DSM from normal and compensated bladders (data not shown). This confirmed that PDBu in- duces bladder smooth muscle contraction through the PKC pathway.

\section{Alterations in the Expression of PKC and the Phosphorylation of CPI-17}

PDBu induces DSM contraction through the PKC-mediated signal transduction pathway. PKC phosphorylates CPI-17. The phosphorylated CPI-17 inhibits myosin phosphatase, raising the level of myosin light chain phosphorylation. The differential $\mathrm{PDBu}$ contraction by DSM from compensated and decompensated bladders indicates that $\mathrm{PBOO}$ may alter the PKC pathway differentially.

To examine changes in the PKC pathway, we determined the expression of two key factors (PKC and CPI-17) and the phosphorylation of CPI-17. Figure 3 shows a representative 


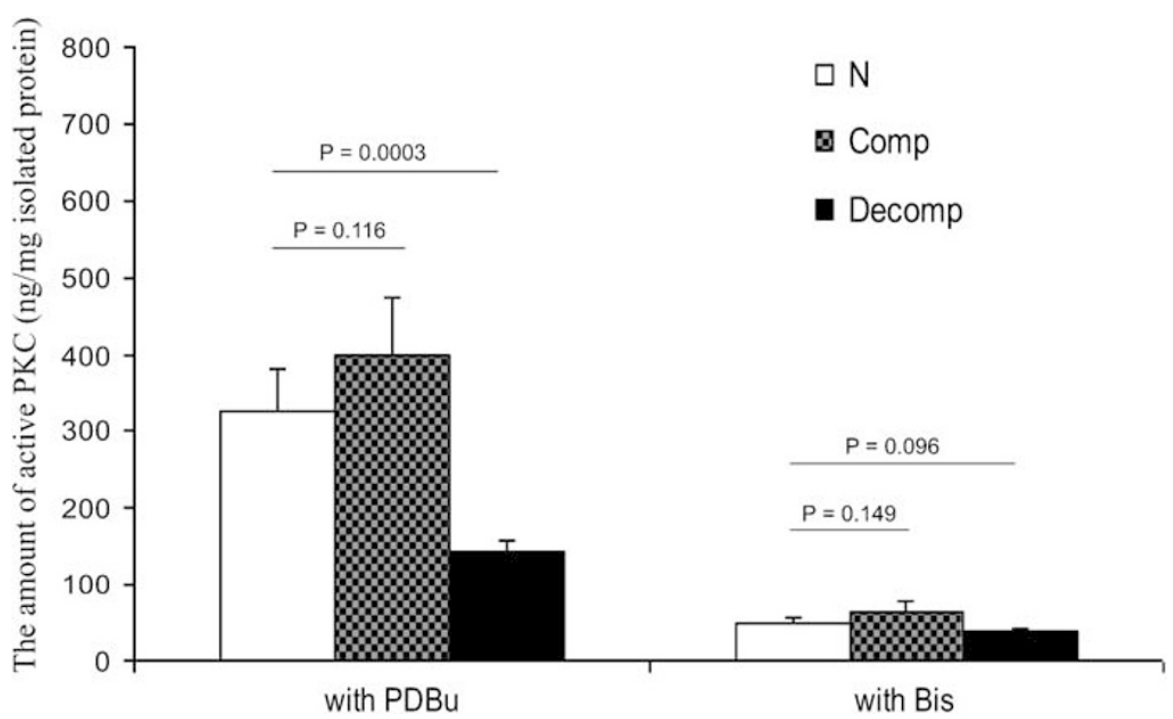

Figure 4 Comparison of PKC activity in normal, compensated and decompensated bladders. PKC activity in DSM from normal, compensated and decompensated bladders was measured using a PKC assay kit (Promega) in the presence of PDBu or Bis. Purified PKC protein was used as standard in the assay. Therefore, the unit of PKC activity is expressed as the amount of purified PKC (ng) per mg isolated protein from different sample. There is a significant decrease in PKC activity in the decompensated bladder DSM compared to normal or compensated bladders $(n=4, P<0.01)$. PKC activity was inhibited in the presence of PKC inhibitor (Bis) in all groups.

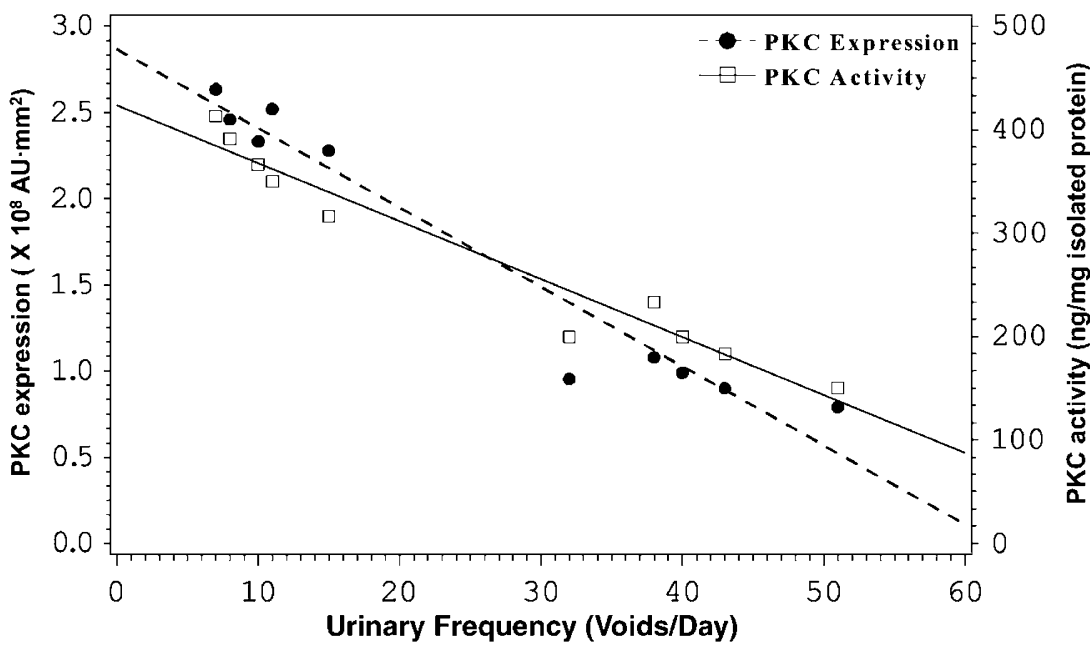

Figure 5 The correlation between PKC function (the expression of $\alpha$ isoform and PKC activity) and urinary frequency. The expression of PKC- $\alpha$ and PKC activity was plotted against urinary frequency. Both plots show an increased urinary frequency is correlated with a decreased PKC function.

western blot image and quantification analysis for the target proteins. Smooth muscle-specific $\alpha$-actin was used as an internal control because our previous study showed that $\mathrm{PBOO}$ did not alter $\alpha$-actin expression at either the mRNA or protein level. ${ }^{19}$ The expression of PKC- $\alpha$ was significantly decreased in decompensated bladder compared with normal and compensated bladders. However, there were no significant differences in CPI-17 expression in DSM among decompensated, normal and compensated bladders. There was no significant difference in phosphor-CPI-17 expression between normal and compensated bladders. However, de- compensated bladder DSM showed a significantly reduction in the amount of phospho-CPI-17. The decreased phosphorCPI-17 in decompensated DSM leads to less myosin light chain phosphorylation that may contribute to less $\mathrm{PDBu}$ contraction.

\section{Alterations of PKC Activity in DSM Induced by PBOO}

Western blot analysis revealed a significant decrease in PKC expression and the phosphorylation of CPI-17 in the DSM from decompensated bladders indicating a decreased PKC activity. To quantify the change of PKC activity, we measured 
PKC activity in the presence of the PKC agonist PDBu or the PKC inhibitor Bis in DSM extracts prepared from normal, compensated and decompensated bladders (Figure 4) using PKC assay kit. Purified PKC protein was used as standard and the unit of PKC activity is expressed as ng purified PKC protein per $\mathrm{mg}$ isolated protein from tissue. There was a significant decrease in PKC activity in decompensated compared with compensated or normal DSM. The level of decreased enzyme activity is associated with the decrease in protein expression, which indicates that decreased enzyme activity is due to decreased protein expression. PKC activity was inhibited by Bis, confirming again that $\mathrm{PDBu}$ stimulation is mediated by PKC.

\section{Correlation between PKC Function and Bladder Function} Figure 5 shows that both PKC- $\alpha$ expression and PKC activity are correlated with urinary frequency. The decrease of PKC expression or activity in $\mathrm{PBOO}$ animal is accompanied by the increase of urinary frequency. This result indicates that PKC function is critical to bladder function. Impaired PKC function is correlated with bladder decompensation.

\section{Immunostaining of PKC and CPI-17 in Bladder Sections from Normal, Compensated and Decompensated Rabbits}

Paraffin sections from normal, compensated and decompensated bladders were prepared for confocal immunofluorescence microscopy. Representative images of the localization of PKC- $\alpha$ (top panels), CPI-17 (middle panels) and phospho-CPI-17 (lower panels) are shown in Figure 6. Images were taken under the identical conditions. The intensity of fluorescent staining is related to the amount of target protein. Decompensated bladder sections showed weak PKC- $\alpha$ and phospho-CPI-17 staining compared to normal and compensated bladder sections. This supports the hypothesis that downregulation of the PKC pathway has a role in bladder dysfunction. CPI-17 immunostaining levels were not remarkably different among the three groups. PKC- $\alpha$ and phosphor-CPI-17 were primarily expressed in the smooth muscle bundle area, especially around smooth muscle cell membranes. CPI-17 was located in both smooth muscle cytoplasm and cell membrane.

\section{DISCUSSION}

$\mathrm{BPH}$ induces significant alterations in the morphology and physiology of the urinary bladder wall. We have studied rabbit model of PBOO to examine the mechanism involved in altered bladder contractility that contributes to impaired bladder function. Normal bladder function requires bladder smooth muscle to produce enough force to empty urine quickly and completely. Following PBOO, the DSM undergoes hypertrophy, accompanied by a remarkable alteration in bladder function. Two-week obstructed animals can be placed in metabolic cages to evaluate the effects of PBOO on bladder function. Some rabbits show relatively normal bladder functions because DSM hypertrophy produces more force to overcome the outlet obstruction (compensated group). Other rabbits show slow and incomplete bladder emptying because DSM cannot generate sufficient force despite DSM hypertrophy (decompensated group). Although there are many studies on compensation/decompensation in heart, the information about this issue in bladder is very limited. There are only a few studies from Zderic's group to show that bladder decompensation is highly associated with a loss of sarcoplasmic reticulum function. ${ }^{6,7}$

In the present study, we did side by side comparison of PKC-mediated signaling in compensated and decompensated DSM. PKC is activated by $\mathrm{PDBu}$ and then phosphorylates its downstream effector protein CPI-17. CPI-17, in turn, regulates myosin light chain phosphatase and the phosphorylation level of the myosin light chain, which is a prerequisite for force generation in smooth muscle. ${ }^{20-22}$ Therefore, we first examined $\mathrm{PDBu}$-induced contraction and then we measured the related signals including the expression and activity of PKC expression and the phosphorylation of CPI17. We show that $\mathrm{PBOO}$ induces differential alterations in PKC-mediated DSM contractility. The PKC-mediated signal transduction pathway has an important function in the regulation of smooth muscle contraction through myosin light chain phosphorylation. Force generation occurs through PKC- $\alpha$ translocation. ${ }^{11,12,23-25}$ Compensated DSM can generate normal force in response to PDBu stimulation, whereas decompensated DSM produces limited PDBu contraction (Figure 2). Reduced PDBu contraction is consistent with the finding reported by Moreland's group. ${ }^{16}$ We also blocked PDBU-induced contraction by preincubation of muscle strips with a PKC inhibitor to confirm that PDBuinduced bladder smooth muscle contraction is acting through the PKC pathway. This result indicates that loss of PKC-mediated contraction might be responsible for the bladder dysfunction seen in the decompensated group.

We further investigated the molecular basis of the loss of PKC-mediated contraction in the decompensated bladder. The protein expression of PKC- $\alpha$ and the enzymatic activity of PKC were significantly reduced in the decompensated bladder (Figures 3 and 4). In vascular smooth muscle, both PKC- $\alpha$ and PKC- $\delta$ isoforms phosphorylate CPI- 17 , especially the $\mathrm{PKC}-\delta$ isoform. ${ }^{23}$ There is no change of $\beta$ isoform induced by $\mathrm{PBOO}$ and the expression of $\theta$ and $\zeta$ is very limited in rabbit bladder (data not shown). Our preliminary data show that PKC- $\alpha$ is the major PKC isoform in rabbit DSM. Expression of the PKC- $\alpha$ isoform in the decompensated bladder decreased $\sim 60 \%$ compared to normal bladders. Likewise, PKC activity was reduced $\sim 60 \%$ in the decompensated bladder. Therefore, it is reasonable to conclude that the loss of PKC activity in the decompensated bladder is due, at least in part, to the downregulation of PKC- $\alpha$.

CPI-17 is a protein that has an important function in the calcium sensitization cascade that maintains force in smooth muscles without additional increases in cytosolic $\mathrm{Ca}^{2+} .{ }^{13}$ 


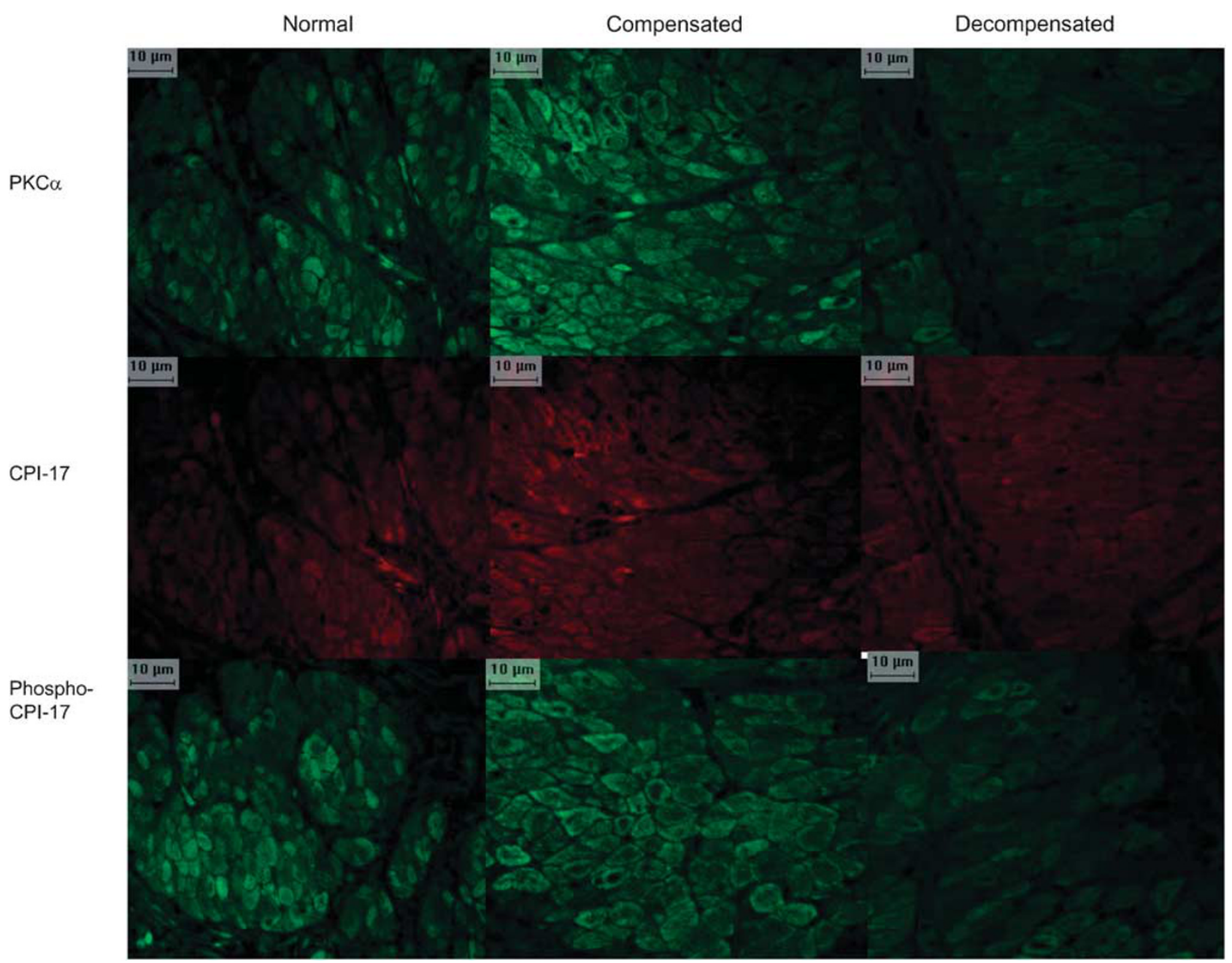

Figure 6 Confocal immunofluorescence micrographs of DSM sections for PKC- $\alpha$, CPI-17 and phospho-CPI-17. Confocal micrographs of bladder sections from normal (left column), compensated (middle column) and decompensated (right column) animals. In situ localization of PKC- $\alpha$ (top panels) and CPI-17 (middle panels) were carried out on same section. Phospho-CPI-17 (lower panes) was prepared on the serial section from the same block. All images were taken using a confocal microscope under the same exposure time and brightness. Negative controls were prepared using preimmune serum instead of the primary antibody (not shown; no signal).

CPI-17 is a downstream effector or PKC. There was a significant decrease in the phosphorylated CPI-17 in the decompensated bladder (Figure 2), although total CPI-17 was not changed. Rho-kinase and PKC are involved in bladder smooth muscle contraction. ${ }^{26,27}$ Rho-kinase has been reported to phosphorylate CPI-17 in vitro. ${ }^{28}$ We have previously shown the overexpression of Rho-kinase in $\mathrm{PBOO}$ DSM. ${ }^{3}$ If Rho-kinase also directly phosphorylates CPI-17, the upregulation of Rho-kinase would be expected to compensate for the downregulation of PKC. However, it appears that Rho-kinase does not increase phospho-CPI-17 level in decompensated DSM. Our finding is consistent with a recent study in vascular smooth muscle demonstrating that Rhokinase does not directly phosphorylate CPI-17 in vivo. ${ }^{29}$ The present study shows that PDBu-induced contraction is associated with the expression of PKC- $\alpha$, PKC activity and CPI-17 phosphorylation. Loss of PDBu-induced contraction in decompensated bladders may result from the downregulation of the PKC-mediated regulatory pathway. Decreased PKC expression leads to a loss of PKC activity and CPI-17 phosphorylation. This loss of CPI-17 phosphorylation leads to low myosin light chain phosphorylation and less force generation. Thus, we show that one of the downstream effectors in the PKC pathway is involved in the regulation of myosin light chain phosphorylation, which is a prerequisite for bladder smooth muscle contraction.

Both compensated and decompensated stages exist in the progress of many diseases but most attention has been paid to heart research. $\mathrm{Na}^{+}-\mathrm{K}^{+}$-ATPase and sarcoplasmic reticulum are well studied in heart failure. The expression of $\mathrm{Na}^{+}-\mathrm{K}^{+}$-ATPase $\alpha_{2}$-isoform is increased in compensated heart whereas it is relatively decreased in decompensated heart in guinea pig. ${ }^{30}$ Sarcoplasmic reticulum proteins show similar bimodal regulation in rat and human heart. ${ }^{31,32}$ 
Although compensation and decompensation in bladder are not life threatening, similar responses to $\mathrm{PBOO}$ are observed in men with BPH. Bladder dysfunction is present in some men with $\mathrm{BPH}$, whereas others have compensated function. $^{33,34}$ The differential responses to $\mathrm{PBOO}$ depicted in the rabbit model provide a better understanding of compensated and decompensated bladder function. Zderic et al show that decreased sarcoplasmic reticulum protein expression is associated with decompensated bladder function and the recovery of bladder function after obstruction reversal is correlated with the expression of ryanodine receptor (one of the sarcoplasmic reticulum protein). ${ }^{6,7}$ We demonstrate that the loss of PKC-mediated contraction and decreased $\mathrm{PKC}$ function occurred in decompensated bladder but not in compensated bladder. The PKC function is correlated with bladder function (Figure 5). The loss of PKC signaling also occurred during the transition of the bladder from compensated to decompensated. Moreover, our preliminary study about $\mathrm{PBOO}$ reversal shows a very interesting result. After removing the urethral ligation, some reversal animals with improved bladder function have improved $\mathrm{PDBu}-$ induced contraction and PKC- $\alpha$ expression, whereas some reversal animals still remain decompensated and there is no increase of PDBu-induced contraction and PKC expression. Our future study will focus on the detailed mechanism for this phenomenon.

In summary, we show that the loss of PDBu-induced DSM contraction and the decreased PKC function correlated with the change in bladder function from compensated to decompensated stage. Therefore, the loss of PKC signaling is one of the underlying mechanisms of bladder dysfunction. This information will be helpful for a better understanding how the bladder becomes decompensated. Thus, a new strategy to manage bladder dysfunction associated with PBOO may be developed based on the better understanding of bladder decompensation.

\section{ACKNOWLEDGEMENTS}

This work was supported by the George O'Brien Urology Research Center grant (P50 DK52620) from the National Institute of Diabetes and Digestive and Kidney Diseases. We thank Dr Moreland for his helpful suggestions and discussions during the course of this study. We thank Jocelyn McCabe for her assistance in the preparation of this manuscript. We also thank Xianqun Luan for his help on data analysis.

1. Levin RM, Longhurst PA, Monson FC, et al. Effect of bladder outlet obstruction on the morphology, physiology, and pharmacology of the bladder. Prostate Suppl 1990:3:9-26.

2. Levin RM, Haugaard N, O'Connor L, et al. Obstructive response of human bladder to BPH vs. rabbit bladder response to partial outlet obstruction: a direct comparison. Neurourol Urodyn 2000;19:609-629.

3. Bing W, Chang S, Hypolite JA, et al. Obstruction-induced changes in urinary bladder smooth muscle contractility: a role for Rho kinase. Am J Physiol Renal Physiol 2003;285:F990-F997.

4. Zhang EY, Stein R, Chang S, et al. Smooth muscle hypertrophy following partial bladder outlet obstruction is associated with overexpression of non-muscle caldesmon. Am J Pathol 2004;164:601-612.
5. DiSanto ME, Stein R, Chang S, et al. Alteration in expression of myosin isoforms in detrusor smooth muscle following bladder outlet obstruction. Am J Physiol Cell Physiol 2003:285:C1397-C1410.

6. Stein R, Gong C, Hutcheson JC, et al. The decompensated detrusor III: impact of bladder outlet obstruction on sarcoplasmic endoplasmic reticulum protein and gene expression. J Urol 2000;164:1026-1030.

7. Stein R, Hutcheson JC, Krasnopolsky L, et al. The decompensated detrusor V: molecular correlates of bladder function after reversal of experimental outlet obstruction. J Urol 2001;166:651-657.

8. Matsumoto T, Nishiyama M, Kobayashi T, et al. Effect of phorbol 12,13dibutyrate on smooth muscle tone in rat stomach fundus. J Smooth Muscle Res 2005:41:107-116.

9. Walsh MP, Andrea JE, Allen BG, et al. Smooth muscle protein kinase C. Can J Physiol Pharmacol 1994;72:1392-1399.

10. Walsh MP, Horowitz A, Clement-Chomienne $O$, et al. Protein kinase $C$ mediation of $\mathrm{Ca}(2+)$-independent contractions of vascular smooth muscle. Biochem Cell Biol 1996;74:485-502.

11. Woodsome TP, Eto M, Everett $A$, et al. Expression of CPI-17 and myosin phosphatase correlates with $\mathrm{Ca}(2+)$ sensitivity of protein kinase $\mathrm{C}$-induced contraction in rabbit smooth muscle. J Physiol 2001;535:553-564.

12. Yoshida M, Nishi K, Machida J, et al. Effects of phorbol ester on lower urinary tract smooth muscles in rabbits. Eur J Pharmacol 1992;222:205-211.

13. Eto M, Senba S, Morita F, et al. Molecular cloning of a novel phosphorylation-dependent inhibitory protein of protein phosphatase-1 (CPI17) in smooth muscle: its specific localization in smooth muscle. FEBS Lett 1997:410:356-360.

14. Eto M, Kitazawa T, Brautigan DL. Phosphoprotein inhibitor CPI-17 specificity depends on allosteric regulation of protein phosphatase-1 by regulatory subunits. Proc Natl Acad Sci USA 2004;101:8888-8893.

15. Somlyo AP, Somlyo AV. Ca2+ sensitivity of smooth muscle and nonmuscle myosin II: modulated by $\mathrm{G}$ proteins, kinases, and myosin phosphatase. Physiol Rev 2003;83:1325-1358.

16. Stanton MC, Austin JC, Delaney DP, et al. Partial bladder outlet obstruction selectively abolishes protein kinase $C$ induced contraction of rabbit detrusor smooth muscle. J Urol 2006;176:2716-2721.

17. Chang S, Hypolite JA, Zderic SA, et al. Enhanced force generation by corpus cavernosum smooth muscle in rabbits with partial bladder outlet obstruction. J Urol 2002;167:2636-2644.

18. Hypolite JA, DiSanto ME, Zheng $Y$, et al. Regional variation in myosin isoforms and phosphorylation at the resting tone in urinary bladder smooth muscle. Am J Physiol Cell Physiol 2001;280:C254-C264.

19. Kim YS, Wang Z, Levin RM, et al. Alterations in the expression of the beta-cytoplasmic and the gamma-smooth muscle actins in hypertrophied urinary bladder smooth muscle. Mol Cell Biochem 1994;131:115-124.

20. Butler TM, Siegman MJ. Control of cross-bridge cycling by myosin light chain phosphorylation in mammalian smooth muscle. Acta Physiol Scand 1998;164:389-400.

21. Dillon PF, Aksoy MO, Driska SP, et al. Myosin phosphorylation and the cross-bridge cycle in arterial smooth muscle. Science 1981;211:495-497.

22. Kamm KE, Hsu LC, Kubota Y, et al. Phosphorylation of smooth muscle myosin heavy and light chains. Effects of phorbol dibutyrate and agonists. J Biol Chem 1989;264:21223-21229.

23. Eto M, Kitazawa T, Yazawa M, et al. Histamine-induced vasoconstriction involves phosphorylation of a specific inhibitor protein for myosin phosphatase by protein kinase C alpha and delta isoforms. J Biol Chem 2001;276:29072-29078.

24. Patil SB, Bitar KN. RhoA- and PKC-alpha-mediated phosphorylation of MYPT and its association with HSP27 in colonic smooth muscle cells. Am J Physiol Gastrointest Liver Physiol 2006;290:G83-G95.

25. Weng TI, Chen WJ, Liu SH. Bladder instillation of Escherichia coli lipopolysaccharide alters the muscle contractions in rat urinary bladder via a protein kinase C-related pathway. Toxicol Appl Pharmacol 2005;208:163-169.

26. Durlu-Kandilci NT, Brading AF. Involvement of Rho kinase and protein kinase $C$ in carbachol-induced calcium sensitization in betaescin skinned rat and guinea-pig bladders. Br J Pharmacol 2006;148:376-384.

27. Ratz $\mathrm{PH}$, Meehl JT, Eddinger TJ. RhoA kinase and protein kinase $\mathrm{C}$ participate in regulation of rabbit stomach fundus smooth muscle contraction. Br J Pharmacol 2002;137:983-992. 
28. Koyama M, Ito M, Feng J, et al. Phosphorylation of CPI-17, an inhibitory phosphoprotein of smooth muscle myosin phosphatase, by Rho-kinase. FEBS Lett 2000;475:197-200.

29. Seko $T$, Ito $M$, Kureishi $Y$, et al. Activation of RhoA and inhibition of myosin phosphatase as important components in hypertension in vascular smooth muscle. Circ Res 2003;92:411-418.

30. Trouve $\mathrm{P}$, Carre $\mathrm{F}$, Belikova I, et al. $\mathrm{Na}(+)-\mathrm{K}(+)$-ATPase alpha(2)-isoform expression in guinea pig hearts during transition from compensation to decompensation. Am J Physiol Heart Circ Physiol 2000;279: H1972-H1981.

31. Arai M, Suzuki T, Nagai R. Sarcoplasmic reticulum genes are upregulated in mild cardiac hypertrophy but downregulated in severe cardiac hypertrophy induced by pressure overload. J Mol Cell Cardiol 1996;28:1583-1590.

32. Hasenfuss $G$, Reinecke $H$, Studer $R$, et al. Relation between myocardial function and expression of sarcoplasmic reticulum $\mathrm{Ca}(2+)$-ATPase in failing and nonfailing human myocardium. Circ Res 1994;75:434-442.

33. Steele GS, Sullivan MP, Sleep DJ, et al. Combination of symptom score, flow rate and prostate volume for predicting bladder outflow obstruction in men with lower urinary tract symptoms. J Urol 2000;164:344-348.

34. Sullivan MP, Yalla SV. Detrusor contractility and compliance characteristics in adult male patients with obstructive and nonobstructive voiding dysfunction. J Urol 1996;155:1995-2000. 